\title{
HÁJEK-RÉNYI-TYPE INEQUALITIES AND LAWS OF LARGE NUMBERS FOR $L_{q}$-MIXINGALE ARRAY
}

\author{
Wang Xuejun, Hu Shuhe, Yang Wenzhi And Shen Yan
}

\begin{abstract}
In the paper, we get the precise results of maximal inequalities and Hájek-Rényi-type inequalities for the partial sums of $L_{q}$-mixingale array $(q>1)$, which generalize and improve the results of Theorem 1 and Theorem 2 in Meng and Lin [8]. We also get the strong law of large numbers, strong growth rate and the integrability of supremum for $L_{q}$-mixingale sequence $(q>1)$, which generalize and improve the results of Corollary 1 in the above cited reference and Corollary 2 in Hansen [4]. At last, a weak law of large numbers for $L_{q}$-mixingale array $(q \geqslant 2)$ is given.
\end{abstract}

Mathematics subject classification (2010): 60E10, 60F15.

Keywords and phrases: Maximal inequality, Hájek-Rényi-type inequality, strong law of large numbers, growth rate, integrability of supremum, mixingale.

\section{REFERENCES}

[1] D. W. K. ANDREWs, Laws of large numbers for dependent non-identically distributed random variables, Econometric Theory, 4 (1988), 458-467.

[2] I. FAZEKAS, AND O. KLESOV, A general approach to the strong law of large numbers, Theory Probab. Appl., 45 (2001), 436-449.

[3] P. Hall, And C. C. Heyde, Martingale Limit Theory and Its Application, Academic Press, New York, 1980.

[4] B. E. Hansen, Strong laws for dependent heterogeneous processes, Econometric Theory, 7 (1991), 213-221.

[5] S. H. Hu, Some new results for the strong law of large numbers, Acta Mathematica Sinica, 46 (2003), 1123-1134. (In Chinese.)

[6] S. H. Hu, G. J. Chen, AND X. J. WANG, On extending the Brunk-Prokhorov strong law of large numbers for martingale differences, Statist. Probab. Lett., 78 (2008), 3187-3194.

[7] D. L. MCLEISH, A maximal inequality and dependent strong laws, Ann. Probab., 3 (1975), 829-839.

[8] Y. J. MENG, AND Z. Y. LIN, Maximal inequalities and laws of large numbers for $L_{q}$-mixingale array, Statist. Probab. Lett., 79 (2009), 1539-1547. 